\title{
Corotating Interaction Regions as Seen by the STEREO Heliospheric Imagers 2007-2010
}

\author{
T.M. Conlon ${ }^{1,2}$ • S.E. Milan ${ }^{2}$ J J.A. Davies ${ }^{3}$. \\ A.O. Williams ${ }^{2}$
}

Received: 18 December 2014 / Accepted: 8 August 2015 / Published online: 10 September 2015

(C) The Author(s) 2015. This article is published with open access at Springerlink.com

\begin{abstract}
NASA's Solar Terrestrial Relations Observatory (STEREO) mission has coincided with a pronounced solar minimum. This allowed for easier detection of corotating interaction regions (CIRs). CIRs are formed by the interaction between fast and slow solarwind streams ejected from source regions on the solar surface that rotate with the Sun. High-density plasma blobs that have become entrained at the stream interface can be tracked out to large elongations in data from the Heliospheric Imager (HI) instruments onboard STEREO. These blobs act as tracers of the CIR itself such that their HI signatures can be used to estimate CIR source location and radial speed. We estimate the kinematic properties of solar-wind transients associated with 40 CIRs detected by the HI instrument onboard the STEREO-A spacecraft between 2007 and 2010. We identify in-situ signatures of these transients at L1 using the Advanced Composition Explorer (ACE) and compare the in-situ parameters with the HI results. We note that solar-wind transients associated with CIRs appear to travel at or close to the slow solar-wind speed preceding the event as measured in situ. We also highlight limitations in the commonly used analysis techniques of solar-wind transients by considering variability in the solar wind.
\end{abstract}

Keywords Corotating interaction regions $\cdot$ Solar wind

\section{Introduction}

Interaction regions are formed in the solar wind by the interaction between fast and slow solar-wind streams from the Sun. When faster material catches up with slower material, previously emitted along the same solar radial, a density enhancement forms at the stream

\section{T.M. Conlon}

tmc3@st-andrews.ac.uk

1 Present address: School of Mathematics and Statistics, University of St. Andrews, St. Andrews, Fife, UK

2 RSPPG, Department of Physics and Astronomy, University of Leicester, Leicester, LE1 3LY, UK

3 RAL Space, STFC Rutherford Appleton Laboratory, Harwell Oxford, Didcot, OX11 0QX, UK 


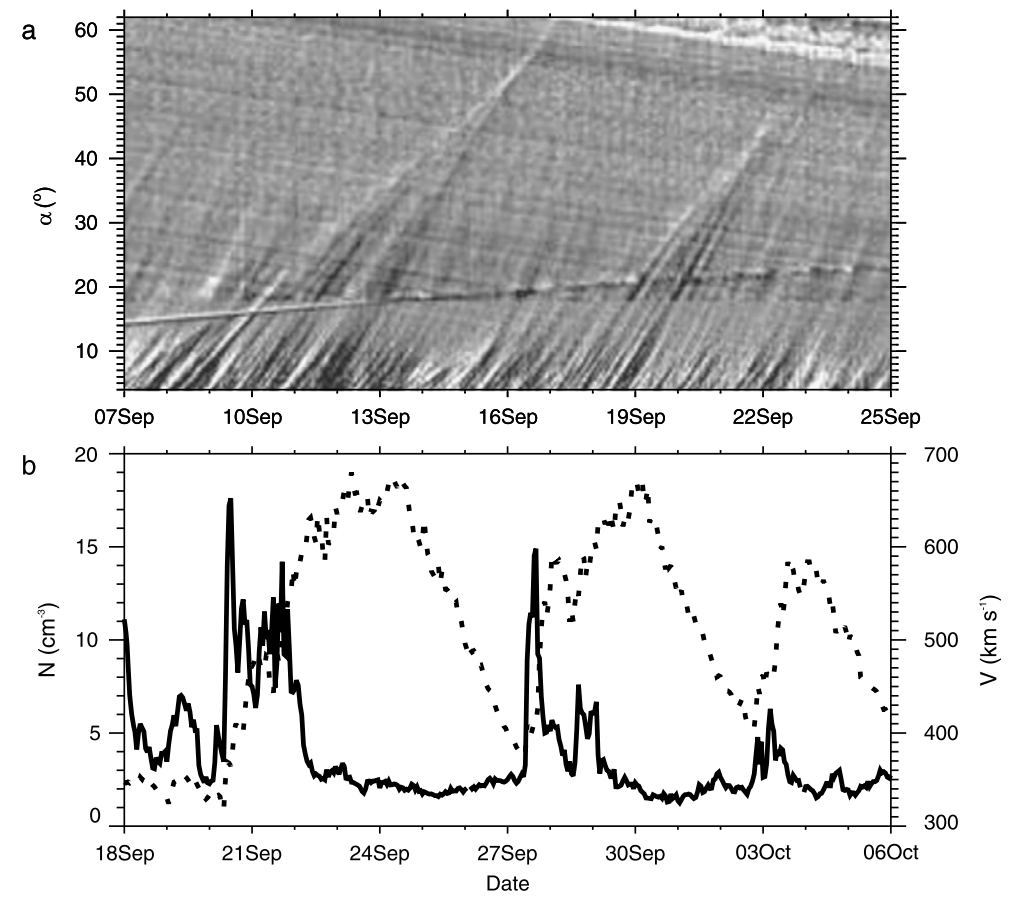

Figure 1 In-situ data from the ACE spacecraft showing proton-number density (solid) and speed (dashed) associated with a pair of CIRs. The denser material can be clearly seen to travel at a speed close to that of the slow solar wind, being followed by more rarefied, faster material. Reproduced with permission from Rouillard et al. (2008).

interface as the faster and slow material originate from different regions of the Sun, so they are threaded by different magnetic-field lines and then cannot mix or flow past one another. This interaction region, which has the overall structure of an Archimedean spiral, is called a corotating interaction region (CIR: Gosling and Pizzo, 1999). Following this density enhancement is a rarefied region consisting of the faster solar-wind material. The faster solar-wind material will be less dense than the slower material as the ions move out faster; thus there is a larger spacing and hence less dense material. Thus, the faster solar wind that has not yet collided with the slower solar wind forms a less dense region following the density enhancement as the feature corotates with the Sun. It is possible that the stream interfaces can form into shocks further out in the heliosphere (Smith and Wolfe, 1976). These features, for a pair of CIRs, and associated high-speed streams in 2007, can be seen in Figure 1 from Rouillard et al. (2008), which presents in-situ data from the Advanced Composition Explorer (ACE) spacecraft taken between 18 September and 6 October 2007. Proton-number density is shown by the solid-black line and the speed by the dashed line. In this figure, the density enhancement typical of a CIR is seen to coincide with solar-wind material appearing to have a speed close to the slow solar wind, which is about $350 \mathrm{~km} \mathrm{~s}^{-1}$ as measured at $1 \mathrm{AU}$ (Lang, 2001).

The source regions of the slow and fast solar wind are thought to differ, with the slower material tending to emanate from regions of closed magnetic field and fast material from regions of open magnetic field (Poletto, 2013). As demonstrated by the Ulysses mission, the fast/slow solar-wind source regions are more clearly delineated during solar minimum and 
hence the deeper solar minimum at the end of Solar Cycle 23 (2007-2010) provides a good opportunity to make CIR observations, as stated by, e.g. Williams et al. (2011). Borovsky and Denton (2006) compared the influences of coronal mass ejections (CMEs) and CIRs on infrastructure and established that, while CMEs pose a greater problem for ground-based systems, CIRs cause greater spacecraft charging and so can have a larger impact on spacebased systems. It is thus important to be able to establish the propagation characteristics of solar-wind transients that form CIRs and forecast their arrival at Earth, especially during the declining phase of the solar cycle, when their influence is the greatest (Davis et al., 2012).

Coronagraphs have been used for decades to image sunlight that has been Thomson scattered from plasma-density enhancements in the solar wind. Observations from such instruments do not extend far out from the Sun (a maximum of $30 \mathrm{R}_{\odot}$ ). More recently, instruments have been developed that allow the solar wind to be imaged out to much greater distances of $1 \mathrm{AU}$ and beyond. An example of such a heliospheric-imaging instrument was the Solar Mass Ejection Imager (Eyles et al., 2003) onboard the NRL/NASA Coriolis spacecraft. This was followed in 2006 by the Heliospheric Imagers (HIs) onboard the two spacecraft of NASA's Solar Terrestrial Relations Observatory (STEREO: Kaiser et al., 2007) mission. Each STEREO spacecraft also hosts a pair of coronagraphs.

Features have been detected using the HI instruments onboard STEREO and the Solar Mass Ejection Imager (SMEI) onboard the Coriolis spacecraft (Tappin and Howard, 2009), which have been associated with CIRs (Rouillard et al., 2008, 2009, 2010; Sheeley et al., 2008). It is thought that these features correspond to blobs of plasma that have become entrained at the stream interface (Rouillard et al., 2008, 2010). Investigations into these features have tended to focus on individual CIR events (Rouillard et al., 2008, 2010) and, while there is undoubtedly great value in doing so, it is also useful to consider the average properties over a wider range of events to look for trends and common features. That is the aim of this study. We shall make some comparisons with Davis et al. (2012) who performed an analysis on a larger number of CIR-associated solar-wind transients.

\section{Remote CIR Observations from STEREO}

The STEREO mission was launched in late 2006 with the particular aim of tracking CMEs as they propagate all the way from the Sun through the heliosphere out to $1 \mathrm{AU}$ and beyond, especially those directed towards the Earth. The mission comprises two spacecraft, each in Earth-like heliocentric orbits, with STEREO-A orbiting ahead of the Earth in its orbit and STEREO-B behind.

The Heliospheric Imagers (HI-1 and HI-2: Eyles et al., 2009) form part of STEREO's Sun Earth Connection Coronal and Heliospheric Investigation (SECCHI: Howard et al., 2008 and Eyles et al., 2009), along with two coronagraphs (COR1 and COR2) and an extreme ultraviolet imager (EUVI). The SECCHI package comprises the mission's remoteimaging capabilities. Taken alone, the HI instruments provide a circular field of view centred on the Ecliptic plane and extending from 4.0 to $88.7^{\circ}$ elongation from the spacecraftSun line. This enables solar-wind transients to be tracked from the corona out to Earth-like distances and beyond. As the STEREO mission progresses, each of the two spacecraft separates from the Sun-Earth line by about $22.5^{\circ}$ per year. This provides SECCHI with an ever changing, stereoscopic view of the region of space between the Earth and Sun. The HI instruments themselves are visible-light cameras, which detect sunlight that has been Thomson scattered from electrons in the solar wind (Eyles et al., 2009). The densities associated with solar-wind transients are relatively low and so, in order to highlight them in the HI data, 

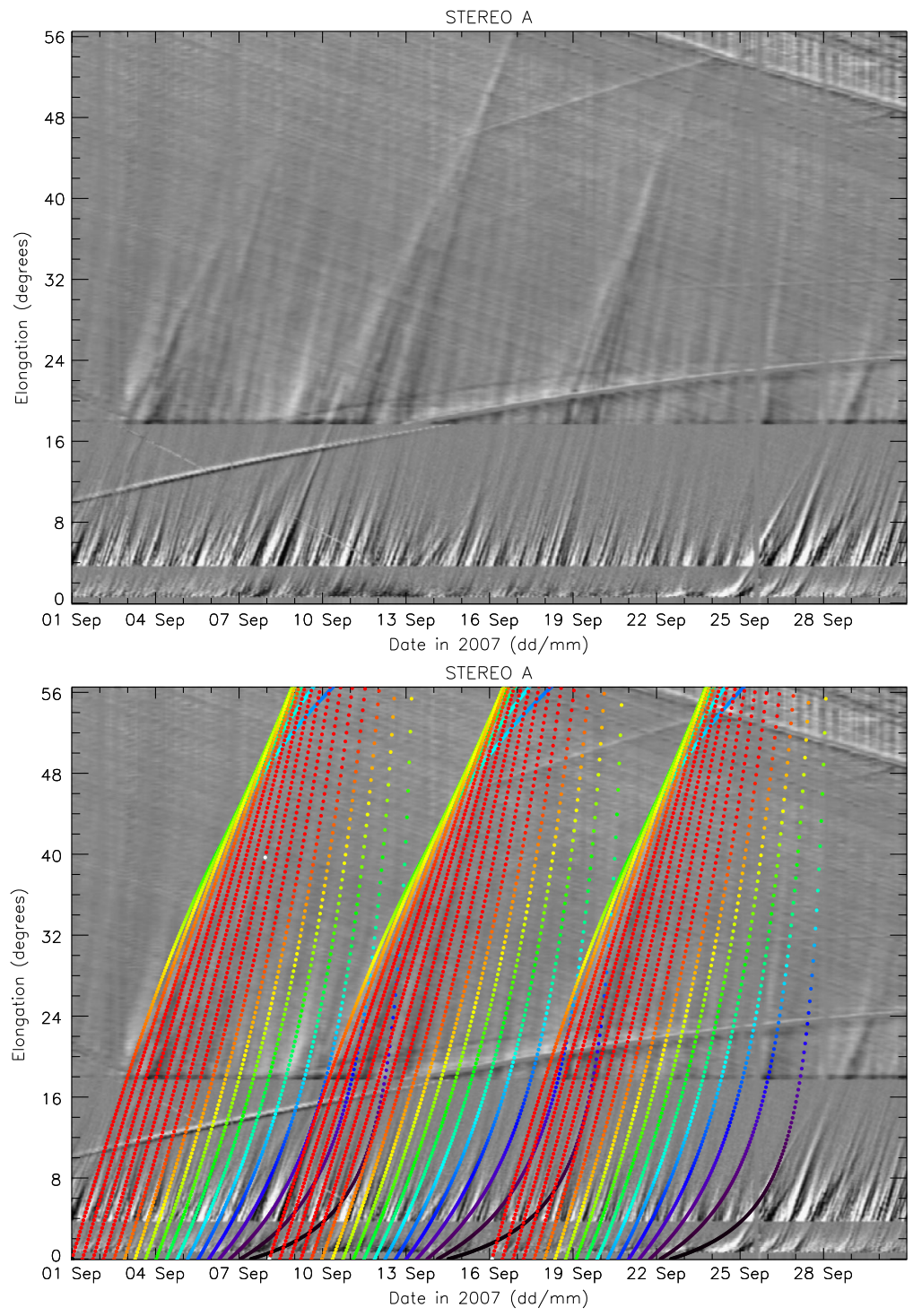

Figure 2 The top panel shows an example J-map extending over September 2007 showing the structure of three CIRs as seen from STEREO-A. Bottom panel shows the same J-map, but with superimposed traces of $\epsilon$ as a function of time, each trace corresponding to a single transient with a constant $\phi$ (see Equation (1)). The traces are colour-coded according to the scattering angle with $\gamma$ going from 0 to $180^{\circ}$ such that those regions in red correspond to a scattering angle of $90^{\circ}$ and should appear brighter than those that are blue/purple, and they have a $\gamma$ close to 0 or $180^{\circ}$.

running-difference images are often used (see Davies et al., 2009 for more information). Moreover, strips along a particular solar radial can then be extracted and a time-elongation map (commonly called a J-map) formed (Sheeley et al., 1999; Davies et al., 2009). An example J-map can be seen in the top panel of Figure 2. This includes data taken from HI-1, HI-2, and COR2 on STEREO-A and encompasses September 2007, showing up to taken 
Figure 3 Schematic showing the geometry when observing CIRs with STEREO-HI. A series of plasma elements are seen moving out from the Sun [S] of which we consider one $[\mathrm{P}]$. This element moves radially outward from the Sun with constant speed $V_{\mathrm{r}}$. Elongation $[\epsilon]$ and propagation angle $[\phi]$ are as shown. The observer, STEREO-A in this instance is at the point labelled A.

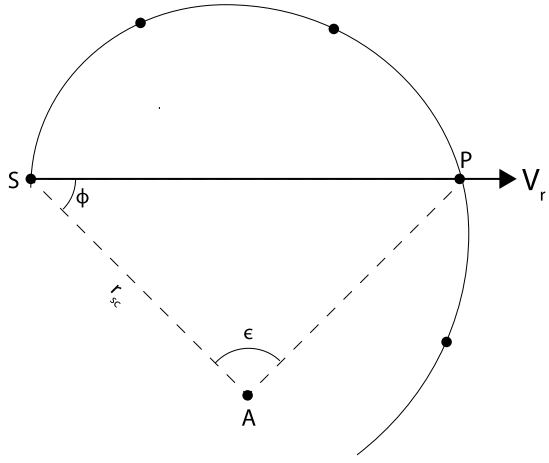

from the Ecliptic plane. There are algorithms designed to remove the stellar background, although they often leave some residue behind. The background F-corona is removed by taking a sequence of images (at least three) and finding the minimum value occurring in each pixel across the images. By removing this, most of the background should be removed. For some larger features, such as the Milky Way, a larger sequence of images might be required. All of this image preparation can be done via the secchi_prep routine, supplied as a part of the Solarsoft package. This figure shows three families of transients, each associated with a CIR. Each CIR is apparent as a family of discrete time-elongation tracks, seen in the figure, that converge at larger elongation values. These transient families have been identified as the HI-2A signatures of CIRs by Rouillard et al. (2008). The time-elongation profile of a solarwind transient, which can be extracted from a J-map, can be analysed to yield an estimate of its radial speed and propagation direction (Howard et al., 2006; Rouillard et al., 2008; Lugaz, Vourlidas, and Roussev, 2009; Davies et al., 2013).

We briefly outline how the kinematic characteristics of features associated with CIRs can be derived from time-elongation profiles using single-spacecraft fitting techniques. Figure 3 shows an example geometry from the STEREO mission. We consider a plasma element [P], characterised as a point, propagating out from the Sun [S] at a fixed propagation angle $[\phi]$ relative to the observer. As the feature propagates out across the HI field of view (HI FOV not indicated), its elongation angle $[\epsilon]$ (the angle between the Sun-spacecraft line and spacecraft-plasma element line) increases. By applying the sine rule to triangle ASP it is possible to arrive at an expression for the elongation variation $[\epsilon(t)]$, as viewed from a single vantage point (Rouillard et al., 2008):

$$
\epsilon(t)=\arctan \left(\frac{V_{\mathrm{r}} t \sin \phi}{r_{\mathrm{SC}}(t)-V_{\mathrm{r}} t \cos \phi}\right),
$$

where $t$ is the travel time from launch (considered for ease to be 0 at $\epsilon=0$ ) of the plasma element, $r_{\mathrm{SC}}$ is the distance from the Sun of the observing spacecraft, and $V_{\mathrm{r}}$ the solarwind transient radial propagation speed. This expression assumes that the propagation of the plasma is at a constant speed and direction. It is possible to fit time-elongation profiles using Equation (1) in order to extract the best-fit values of $V_{\mathrm{r}}$ and $\phi$ that correspond to that profile (Rouillard et al., 2008). This technique is termed fixed- $\phi$ fitting (Kahler and Webb, 2007). A single CIR as imaged by HI results in a family of time-elongation profiles, each corresponding to (it is surmised) an individual blob entrained at the stream interface. Due to the geometry of the situation, these tracks will converge at higher elongation values as seen by STEREO-A/HI and diverge at higher elongation values as seen by STEREO-B/HI (Rouillard et al., 2009). There are various techniques for fitting time-elongation profiles; 
Table 1 Trajectories of transients determined from HI-A observations. The events are taken from 2007 and the time is that at which the transient passed the $\epsilon=5^{\circ}$ point. The estimated propagation characteristics obtained by Rouillard et al. (2010) are shows in columns labelled $\phi$ and $V_{\mathrm{r}}$. The final column, labelled New $V_{\mathrm{r}}$ provides an estimate of the radial propagation speed assuming the spacecraft motion is corrected for, arrived at by assuming the findings of Conlon, Milan, and Davies (2014).

\begin{tabular}{llllll}
\hline Track & Date & $\begin{array}{l}\text { Time } \\
{[\mathrm{UT}]}\end{array}$ & $\begin{array}{l}\phi \\
{\left[{ }^{\circ}\right]}\end{array}$ & $\begin{array}{l}V_{\mathrm{r}} \\
{\left[\mathrm{km} \mathrm{s}^{-1}\right]}\end{array}$ & $\begin{array}{l}\text { New } V_{\mathrm{r}} \\
{\left[\mathrm{km} \mathrm{s}^{-1}\right]}\end{array}$ \\
\hline CIR-D & & & & & \\
$\mathrm{a}$ & 09 Sep & 0107 & $84 \pm 04$ & $268 \pm 14$ & 268 \\
$\mathrm{~b}$ & $09 \mathrm{Sep}$ & 1945 & $82 \pm 09$ & $288 \pm 24$ & 288 \\
$\mathrm{c}$ & $10 \mathrm{Sep}$ & 0657 & $78 \pm 10$ & $285 \pm 16$ & 285 \\
$\mathrm{~d}$ & $10 \mathrm{Sep}$ & 2139 & $63 \pm 11$ & $299 \pm 11$ & 298 \\
$\mathrm{e}$ & $11 \mathrm{Sep}$ & 0752 & $61 \pm 11$ & $311 \pm 18$ & 309 \\
$\mathrm{f}$ & $11 \mathrm{Sep}$ & 2019 & $37 \pm 07$ & $335 \pm 07$ & 324 \\
CIR-E & & & & & \\
$\mathrm{g}$ & $17 \mathrm{Sep}$ & 0531 & $80 \pm 08$ & $307 \pm 21$ & 307 \\
$\mathrm{~h}$ & $17 \mathrm{Sep}$ & 1412 & $80 \pm 11$ & $306 \pm 34$ & 306 \\
$\mathrm{i}$ & $18 \mathrm{Sep}$ & 0138 & $59 \pm 10$ & $321 \pm 07$ & 319 \\
$\mathrm{j}$ & $18 \mathrm{Sep}$ & 0720 & $52 \pm 09$ & $319 \pm 07$ & 316 \\
$\mathrm{k}$ & $18 \mathrm{Sep}$ & 1719 & $55 \pm 12$ & $340 \pm 09$ & 337 \\
1 & $19 \mathrm{Sep}$ & 0612 & $40 \pm 06$ & $324 \pm 07$ & 314 \\
\hline
\end{tabular}

however, the fixed- $\phi$ approximation outlined here is more appropriate to solar-wind transients that are point-like in cross section as opposed to spatially extended. As the individual transients that make up a CIR are assumed to be relatively small in size, the fixed- $\phi$ geometry should accurately describe their geometry. Analysis conducted by Rouillard et al. (2008) on transients associated with the CIRs (for which in-situ data can be seen in Figure 1) ascertained propagation speeds of around $270 \mathrm{~km} \mathrm{~s}^{-1}$, lower than the in-situ speed that had been measured. This leads to some ambiguity surrounding the propagation characteristics ascertained from HI J-maps.

As discussed by Conlon, Milan, and Davies (2014), $\phi$ will not actually be constant even if the transient propagates out radially, as spacecraft motion during the propagation of the transient will cause $\phi$ to vary, changing by about $1^{\circ}$ per day. This effect will increase $\phi$ relative to STEREO-A and decrease $\phi$ for STEREO-B. The authors suggest that Equation (1) could be modified into

$$
\epsilon(t)=\arctan \left(\frac{V_{\mathrm{r}} t \sin \left(\phi_{0} \pm \frac{360 \pm 22.5}{n_{\mathrm{y}}} t\right)}{r_{\mathrm{SC}}(t)-V_{\mathrm{r}} t \cos \left(\phi_{0} \pm \frac{360 \pm 22.5}{n_{\mathrm{y}}} t\right)}\right)
$$

to account for this, where $t$ is in seconds and then $n_{\mathrm{y}}$ is the number of seconds in a year. It is important to note that Equation (2) only applies to STEREO observations made in the Ecliptic plane.

Rouillard et al. (2010) used Equation (1) to fit individual time-elongation profiles associated with features within CIRs. They noticed that their estimated radial propagation speed appeared to change as a function of propagation direction $[\phi]$ the two appearing to be anti-correlated when using fixed- $\phi$ fitting. They observed this primarily with STEREO-A, for which they had more data. The effects of spacecraft motion on fixed- $\phi$ fitting of single time-elongation profiles noted by Conlon, Milan, and Davies (2014) change the fitted propagation speed and $\phi$ so we first consider whether it can explain the trend observed by Rouillard et al. (2010).

Table 1 (excluding the final column) reproduces Table 1 from Rouillard et al. (2010) (slightly restructured for ease of reading), showing speed and propagation direction from in- 

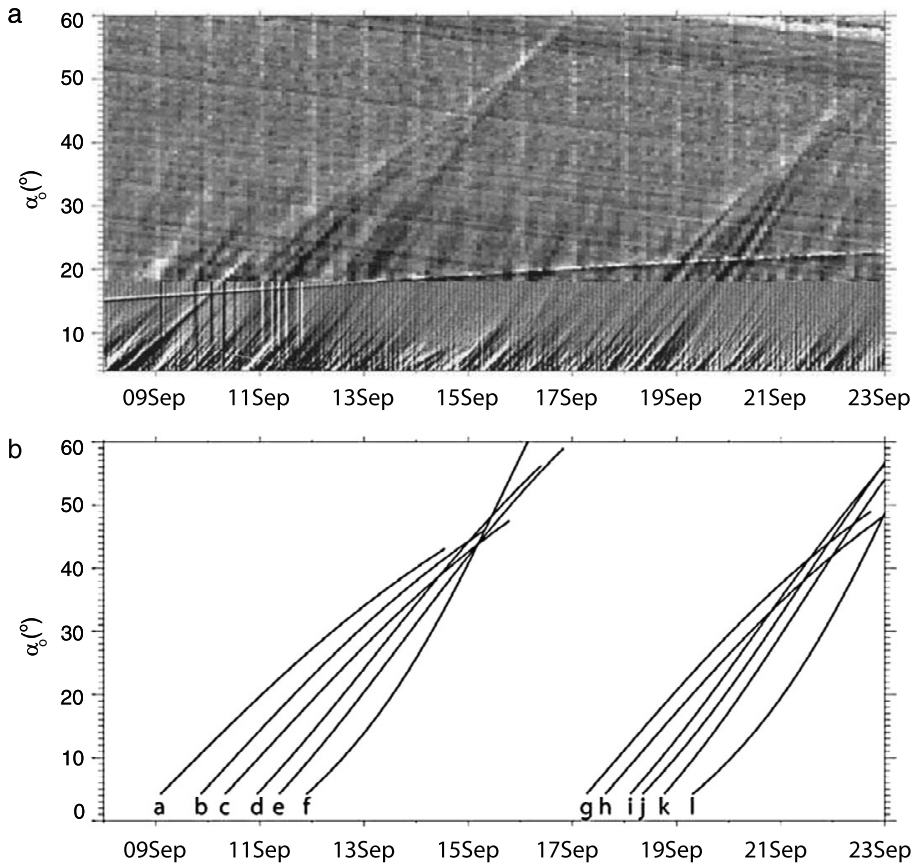

Figure 4 (a) Two CIRs from 2007, with the individual time-elongation profiles shown and labelled in panel b. CIR-D is on the left (associated with tracks a-f) and CIR-E is on the right (associated with tracks $\mathrm{g}-1)$. This figure is reproduced with permission from Rouillard et al. (2010).

dividual features associated with each of two STEREO-A CIRs, labelled CIR-D and CIR-E. Figure 4 (reproduced from Rouillard et al., 2010) shows (a) a time-elongation map covering the two CIRs in question and (b) the individual best-fit time-elongation profiles. The final column of Table 1 gives an estimate for the radial propagation speed assuming that spacecraft motion is corrected for. Although the variation in $\phi$ has not been included here, this was a systematic variation of no more than $2^{\circ}$ in each case, consistent with the results presented by Conlon, Milan, and Davies (2014). Correcting for spacecraft motion makes little difference to the estimated speed. However, for small $\phi$-values, there is a noticeable difference, in each case acting such that the relationship between $\phi$ and $V_{\mathrm{r}}$ previously noted is reduced, although not completely removed; i.e. for lower $\phi$-values the revised speed was lower than that presented by Rouillard et al. (2010). This is consistent with the findings of Conlon, Milan, and Davies (2014). As mentioned previously, Rouillard et al. (2010) also performed this analysis on STEREO-B/HI observations; however, they only have a few tracks from each CIR as seen from STEREO-B and so we have only included the STEREO-A results here.

Most previous studies (such as Rouillard et al., 2008, 2010) have performed fitting on single time-elongation profiles, each corresponding to a single blob entrained at the stream interface; however, Sheeley and Rouillard (2010) fitted the entire family of CIR tracks and we follow their example. By varying $V_{\mathrm{r}}$ and the location of the source, a best fit to an entire family of traces can be achieved by eye. In this way, the fitting is conducted on a family of traces at once, as opposed to individual traces. The lower panel of Figure 2 shows an example of this, with traces fitted to three different families of tracks.

The K-coronal component of the light detected by $\mathrm{HI}$ is sunlight that has been Thomson scattered by electrons in the inner heliosphere. Along any particular line of sight, the HI in- 
strument is most sensitive to light scattered from the intersection of the line of sight with the "Thomson surface", the sphere with a diameter extending from the observer to the Sun. This results in an intensity varying as $\sin ^{2} \gamma$, where $\gamma$ is the scattering angle (Howard and Tappin, 2009), i.e. $\angle$ SPO. This is counterbalanced to some extent by the "Thomson Plateau", the overall result of which is that the scattering brightness is actually approximately constant over a broad range of solar exit angles for a given position in the image plane, i.e. features can still be seen away from the Thomson sphere. We have colour-coded the traces in Figure 2 according to the scattering angle $[\gamma]$. The traces themselves are plotted at $5^{\circ}$ intervals of $\phi, 90^{\circ}$ to $0^{\circ}$ for 400 hourly time steps. The single best-fit speeds for the three CIRs seen in Figure 2 are $280 \mathrm{~km} \mathrm{~s}^{-1}, 280 \mathrm{~km} \mathrm{~s}^{-1}$, and $450 \mathrm{~km} \mathrm{~s}^{-1}$. The two events labelled by Rouillard et al. (2010) as CIR-D and CIR-E have speeds of $280 \mathrm{~km} \mathrm{~s}^{-1}$ derived by this method. These propagation speeds, derived by fitting to the whole family of tracks, mainly fall below the range of fitted speeds for the individual blobs presented by Rouillard et al. (2010) for CIR-D and CIR-E.

\section{CIR Propagation Speed}

40 clear CIRs (i.e. 40 families of converging tracks) were identified in Ecliptic STEREOA/HI J-maps from January 2007 to June 2010. Fits, by eye, were performed to the whole family of traces in each instance to estimate radial propagation speeds, as outlined in Section 1. The fitting was performed to equations that incorporate the correction for spacecraft motion outlined by Conlon, Milan, and Davies (2014). For each CIR identified this way, we thus have a single estimated radial propagation speed for all of the features associated with the CIR (as opposed to a single speed per time-elongation profile). The details of the CIRs can be seen in Table 2, with the first column giving the start time of the elongation profiles that start at $\phi=180^{\circ}$ to the nearest hour, and the second column showing the propagation speed. Profiles of different speeds were tested to see how far from the values presented in Table 2 one could go before the fit was clearly wrong, i.e. before the fitted family of traces was clearly very different from the traces in the underlying J-map. In this manner, it was possible to arrive at an estimate for the uncertainty in the speeds of about $\pm 30 \mathrm{~km} \mathrm{~s}^{-1}$.

Figures 1 and 5 show the in-situ and imaging signatures, respectively, of one of the CIRs considered in this study, being the third event in Figure 2 and second event in Figure 4. The top panel of Figure 5 reproduces a limited part of the J-map over an 11-day period: extending from 16 to 27 September 2007. The bottom panel shows the same J-map, but this time with the traces associated with the current fitting procedure. The curves start at $\phi$ of $180^{\circ}$ and decrease to $0^{\circ}$ in $5^{\circ}$ steps. In this example, the overplotted family of tracks convincingly fits the underlying CIR structure (with the proviso that the fit is poorer at lower elongation values, as noted by Williams et al. (2009)) and yields a radial of speed $450 \mathrm{~km} \mathrm{~s}^{-1}$. The in-situ measured speed of the transient is around $400 \mathrm{~km} \mathrm{~s}^{-1}$.

Figure 6 considers each of the 40 events identified in STEREO-A/HI J-maps and compares the average CIR radial propagation speeds, as determined by fitting to entire families of tracks in the HI J-maps, to the speed of the associated density enhancement as measured in-situ at ACE. These we selected on the basis of them having clear signatures in HI and accompanying solar-wind data at ACE. We predicted the arrival time of features observed in STEREO/HI at the ACE spacecraft to identify in situ signatures. The points in blue disregard spacecraft motion and those in black incorporate it. It can be seen that while there is a large amount of scatter, the HI-derived radial speeds that do not incorporate spacecraft motion are consistently lower than the speeds of the associated density enhancements as 
Table 2 Timing and propagation speed of the 40 events used in this study. The start time is the start time of the time-elongation profile that starts at $\phi=180^{\circ}$ to the nearest hour in the format dd mmm yyyy hh.

\begin{tabular}{|c|c|c|c|}
\hline Start time & $V_{\mathrm{r}} \pm 30\left[\mathrm{~km} \mathrm{~s}^{-1}\right]$ & Start time & $V_{\mathrm{r}} \pm 30\left[\mathrm{~km} \mathrm{~s}^{-1}\right]$ \\
\hline 04 May 2007 09:00 & 290 & 06 Nov 2008 22:00 & 270 \\
\hline 04 June 2007 12:00 & 400 & 25 Nov 2008 16:00 & 360 \\
\hline 10 June 2007 18:00 & 390 & 14 Dec 2008 14:00 & 350 \\
\hline 11 July 2007 02:00 & 360 & 25 Dec 2008 04:00 & 250 \\
\hline 18 July 2007 00:00 & 320 & 10 Jan 2009 07:00 & 330 \\
\hline 28 July 2007 06:00 & 350 & 18 Jan 2009 19:00 & 310 \\
\hline 08 Aug 2007 16:00 & 440 & 29 Jan 2009 00:00 & 340 \\
\hline 25 Aug 2007 00:00 & 280 & 05 Feb 2009 18:00 & 350 \\
\hline 01 Aug 2007 00:00 & 280 & 15 Feb 2009 15:00 & 300 \\
\hline 10 Aug 2007 18:00 & 450 & 28 Feb 2009 15:00 & 330 \\
\hline 28 Sep 2007 10:00 & 280 & 04 Mar 2009 00:00 & 330 \\
\hline 04 Nov 2007 00:00 & 360 & 31 Mar 2009 00:00 & 310 \\
\hline 13 Jan 2008 00:00 & 300 & 24 May 2009 12:00 & 370 \\
\hline 24 Jan 2008 18:00 & 400 & 27 Sep 2009 00:00 & 240 \\
\hline 10 Feb 2008 06:00 & 350 & 22 Oct 2009 08:00 & 260 \\
\hline 18 Feb 2008 06:00 & 320 & 14 Deb 2010 02:00 & 280 \\
\hline 09 Mar 2008 12:00 & 380 & 20 Feb 2010 18:00 & 390 \\
\hline 11 May 2008 08:00 & 390 & 06 May 2010 02:00 & 400 \\
\hline 04 July 2008 04:00 & 320 & 11 May 2010 12:00 & 330 \\
\hline 10 July 2008 06:00 & 340 & 01 June 2010 20:00 & 380 \\
\hline
\end{tabular}

measured in situ at the ACE spacecraft, a trend that is no longer seen once spacecraft motion is incorporated into the analysis. It might be expected that the stream blobs would travel at some intermediate speed between the preceding slow and following fast solar-wind speed, but this does not agree, however, with the findings of Sheeley et al. (1997), who use observations from the Large Angle and Spectroscopic Coronagraph (LASCO) on board the Solar and Heliospheric Observatory (SOHO) to track stream blobs close to the Sun. They noted that the blobs appeared to passively trace the outflow of the slow solar wind, although their observations were not restricted to entrained blobs.

\subsection{CIR Periodicity and Predicted Arrival Times}

If the same long-lived source region on the Sun is causing a repeating sequence of events from the vantage of a single, fixed observer, then it should have a constant Carrington longitude, just separated by one solar-rotation period (assuming the source region remains fixed on the solar surface). Calculating the Carrington longitude should also give an indication of the longevity of a given source region. Knowing the rotation rate of the Sun and the approximate radial propagation speed and direction of a feature associated with the CIR allows one to predict its arrival at any point in the heliosphere, including tracing it back to a source at the Sun. Typical analysis of CIRs makes assumptions which would be invalid if there were significant temporal variability over a few days to a week. Thus, it is of interest to this study.

The top panels of Figures 7 and 8 show year-long time-series of the Carrington longitude for 2008. Each point on the plot corresponds to the location of a particular CIR source region 

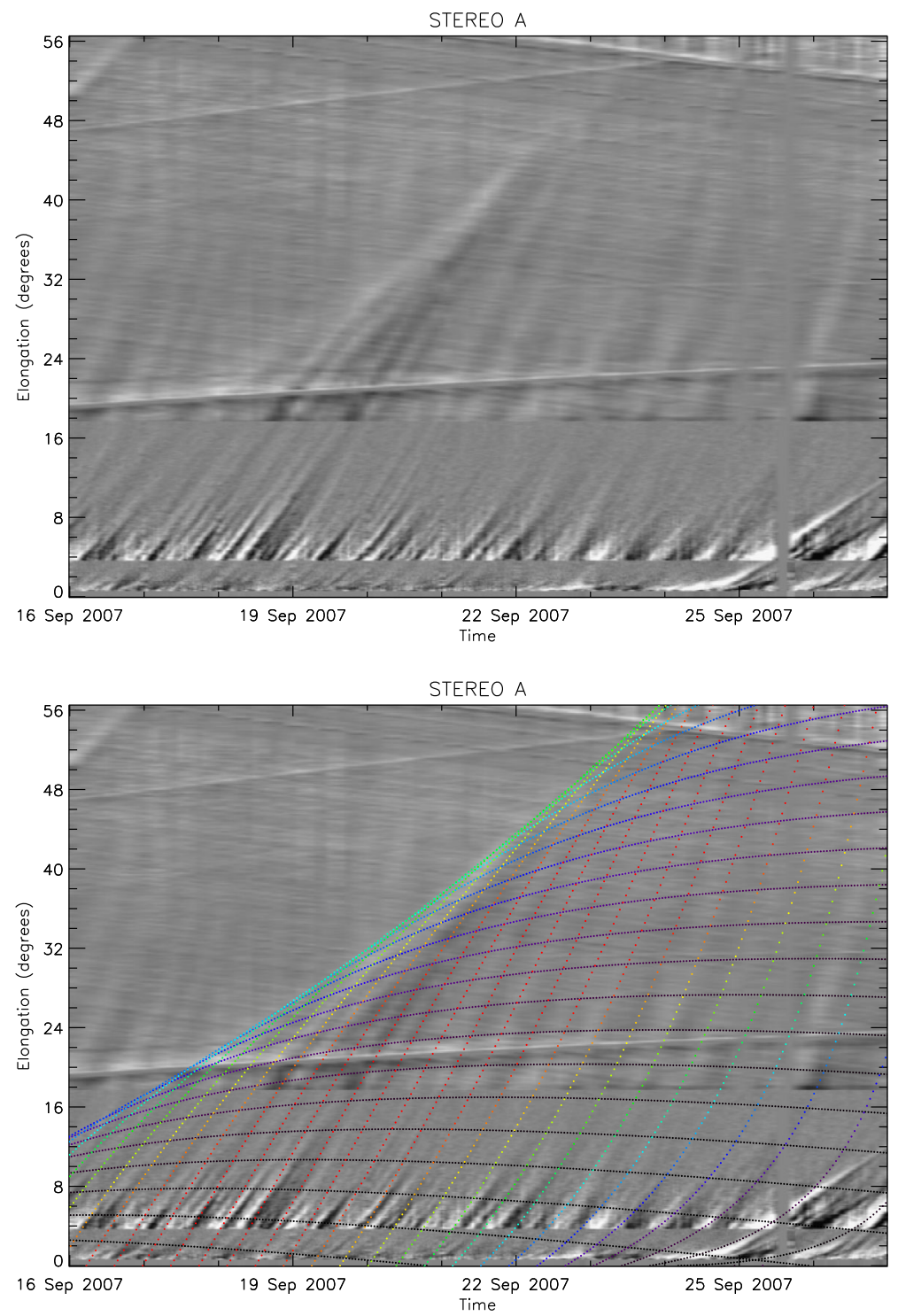

Figure 5 J-map for an 11-day period in September 2007 in which can be seen a CIR (top panel). The bottom panel shows the fitted profiles, overplotted but no longer with constant $\phi$-values. The coloured traces start at $180^{\circ}$ and decrease to $5^{\circ}$ in $5^{\circ}$ increments. These $\phi$-values are the starting values of each trace, but $\phi$ is now allowed to change with time (i.e. each trace does not now have a constant $\phi$-value). Once again, the colour coding is done according to the scattering angle $[\gamma]$ such that one would expect the red areas to be brighter than the blue.

on the Sun as it crosses the sub-solar point as estimated from the J-maps and the verticalpurple-dashed lines show Carrington rotations. The bottom three panels of Figures 7 and 8 show the in-situ solar-wind speed and proton-number density, respectively, as measured at each of STEREO-B, ACE, and STEREO-A. Figure 9 is the same as Figure 7 but for 2009. The in-situ speed and density data are taken from the In-situ Measurement of Particles And 
Figure 6 The CIR speed as measured by HI versus the speed associated with the enhancement in density as measured in-situ by ACE. The blue points show those speeds as estimated from $\mathrm{HI}$ without incorporating spacecraft motion and the black crosses including spacecraft motion. A general increase in propagation speed estimated from $\mathrm{HI}$ can be seen when incorporating spacecraft motion.

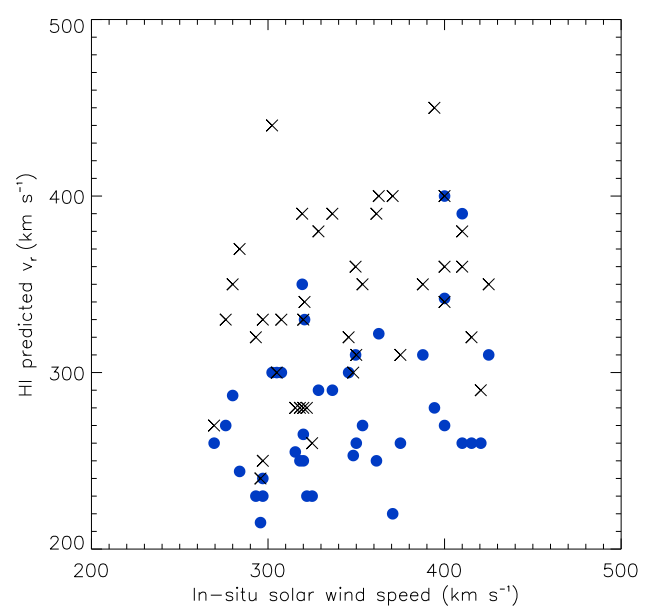

Figure 7 Top panel: The Carrington longitude of CIR source regions as they cross the sub-solar point. Carrington rotations are shown by purple-solid-vertical lines. The lower three panels show the in-situ speed data taken from the STEREO and ACE spacecraft, with predicted CIR arrival times overplotted as red-dashed lines and the speeds estimated from STEREO-HI plotted as blue diamonds. This plot covers 2008.

CME Transients (IMPACT) suite of instruments (Acuña et al., 2008) on the STEREO spacecraft and from NASA's CDAWeb. The vertical-red lines in the in-situ panels correspond to predicted arrival times of each of the CIRs considered in this study at each of these spacecraft. Plots such as this were generated for each of the years used in this study $(2007,2008$, $2009,2010)$. The Carrington longitude is defined to be $349.03^{\circ}$ at 00:00 UT on 1 January 


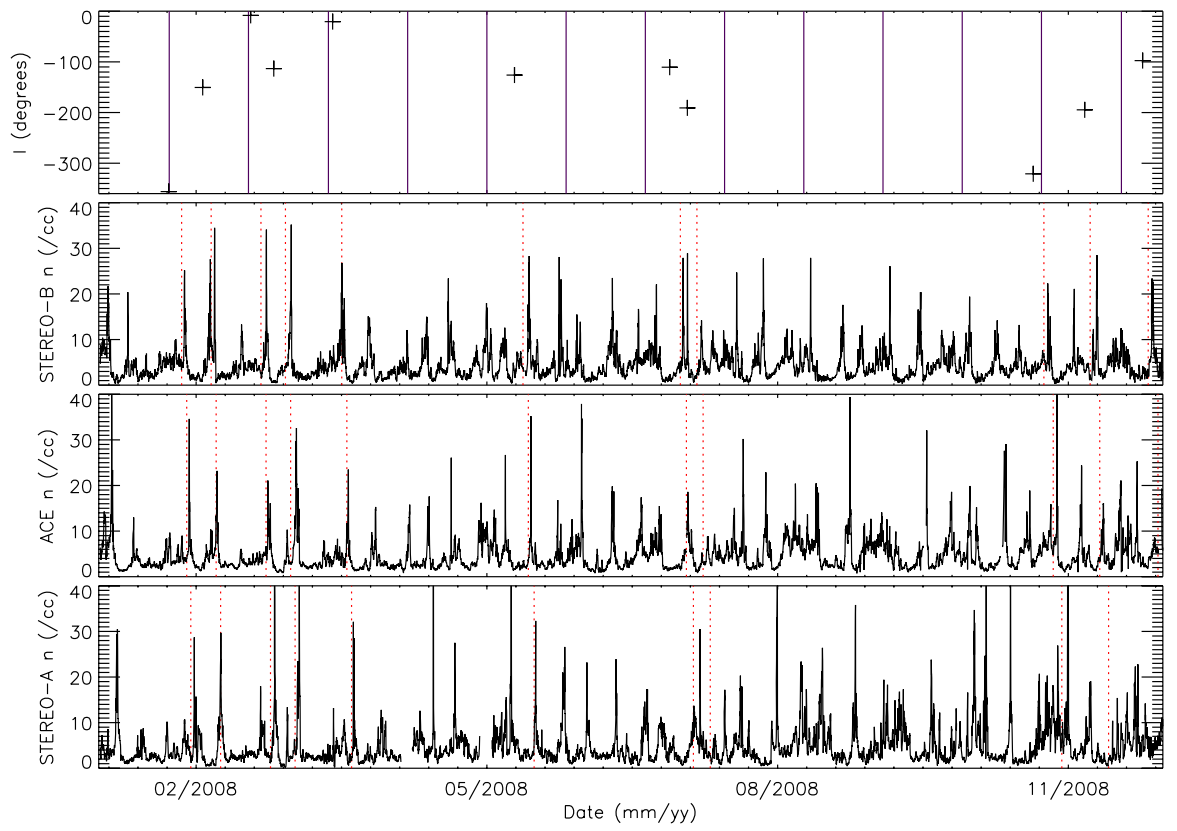

Figure 8 Top panel: The Carrington longitude of CIR source regions as they cross the sub-solar point. Carrington rotations are shown by purple-solid-vertical lines. The lower three panels show the in-situ number density data taken from the STEREO and ACE spacecraft, with predicted CIR arrival times overplotted as red-dashed lines. This plot covers 2008.

1995. Thus, the Carrington longitude [l], as presented in the upper panels of Figures 7, 8 and 9 , is given by

$$
l=\left(329.03-\frac{360 x}{P}\right) \bmod 360,
$$

where $x$ is the number of days that have passed since 00:00 UT 1 January 1995 and $P$ is the synodic rotation period [26.24 days]. In the three Carrington rotations near the start of Figures 7 and 8, which both cover 2008, is a feature, at a Carrington longitude close to $0^{\circ}$, that appears consistently every solar rotation. Another source region appears near a longitude of $130^{\circ}$ for two consecutive solar rotations. The clearest example of a recurring feature can be seen in Figure 9. Here, a series of points labelled "C" can be seen to reoccur at approximately the same Carrington longitude with a periodicity of about one Carrington rotation (other source regions coexist with this). However, the longitude of the source region appears to slowly drift. This would suggest either a source region that does not perfectly corotate with the Sun, or else that the rotation rate that we use for the Sun is not exactly appropriate for the Ecliptic plane. It is also possible that uncertainties with the derived longitudes cause this. Nevertheless, this seems to show that a given source region on the Sun can persist for multiple solar rotations, and considering the associated in-situ speed and density plots it can be seen that, as would be expected, there is considerable variation on these time scales (months). There are also more than one observed feature per solar rotation, so there are multiple source regions on the Sun.

As mentioned previously, knowing the radial propagation speed of features associated with the CIRs observed and the rotation rate of the Sun, it is possible to calculate pre- 


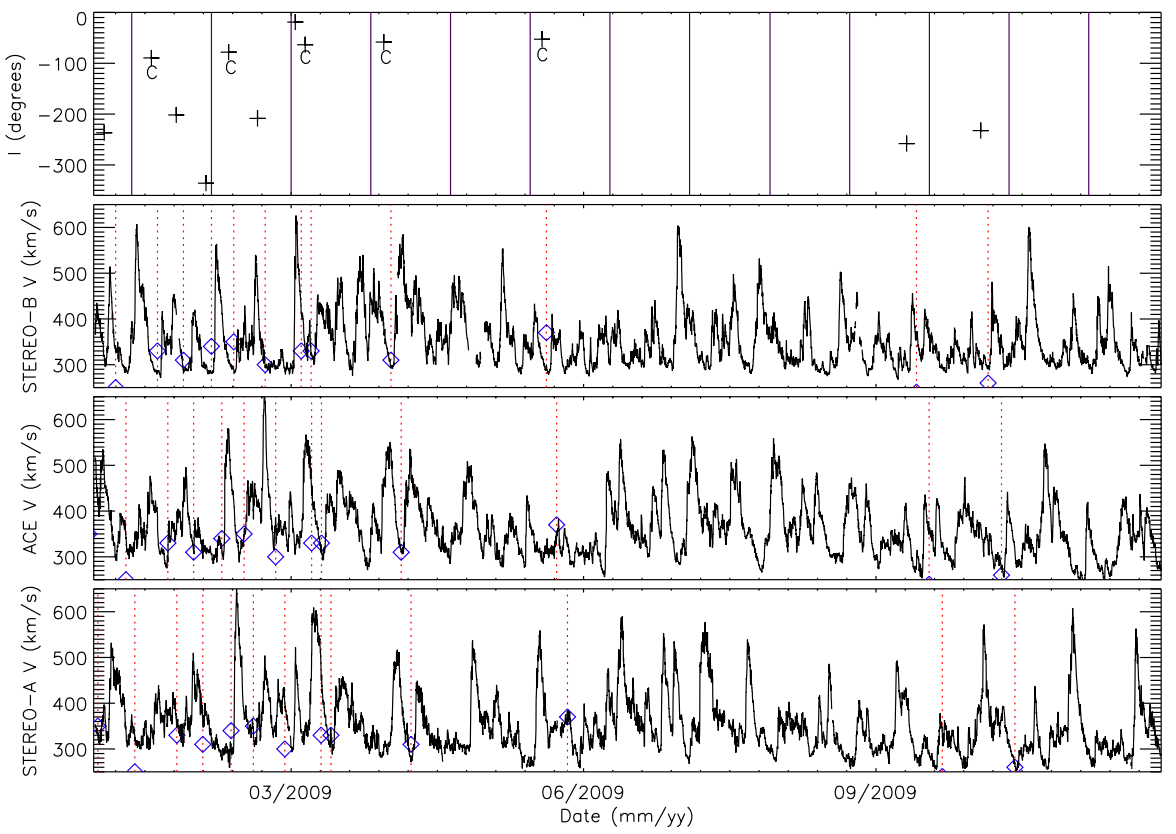

Figure 9 Top panel: The Carrington longitude of CIR source regions as they cross the sub-solar point. Carrington rotations are shown by purple solid vertical lines. The lower three panels show the in-situ speed data taken from the STEREO and ACE spacecraft, with predicted CIR arrival times overplotted as red-dashed lines and the speeds estimated from STEREO/HI plotted as blue diamonds. This plot covers 2009. In the top panel are a series of features labelled as "C" These features appear at a very similar longitude, separated by a single solar rotation, indicating that the stream interface causing them is tied to the same feature at the Sun which is then causing a feature that is seen every solar rotation.

dicted arrival times at various places in the heliosphere. The bottom three panels of Figures 7 and 9 show the in-situ bulk solar-wind speed as measured at STEREO-B, ACE, and STEREO-A for 2007 and 2009, respectively. The vertical-red lines correspond to predicted arrival times of each of the CIRs considered in this study at each of these spacecraft. The bottom three panels of Figure 8 show the corresponding in-situ proton-number density data for 2008. Comparing Figures 7 and 8, we see multiple increases and decreases in solar-wind speed with corresponding density spikes at the leading edge of many of the $V_{\mathrm{sw}}$ increases. Many of these are CIR signatures, as shown in Figure 1. The same CIRs will, in general, be seen at each spacecraft, although staggered in time due to the observers' different heliospheric longitudes. Near the start of 2008, the spacecraft are close in longitude and the offset in arrival time of solar-wind features at the different spacecraft is small, but this increases as the STEREO spacecraft drift away from the Sun-Earth line.

In general there is good correspondence between the predicted arrival of a CIR derived from the STEREO-A/HI data at an observing spacecraft and the arrival time of a density enhancement in-situ at that spacecraft, as also observed by Williams et al., 2011. The propagation speed of each event, estimated from HI, is shown by the blue diamonds in Figures 7 and 9 and shows the consistency with the slow solar-wind speed as described in Section 3. It can be seen, by looking at Figures 7 and 9, that the arrival times match well with the slow solar-wind speed. 


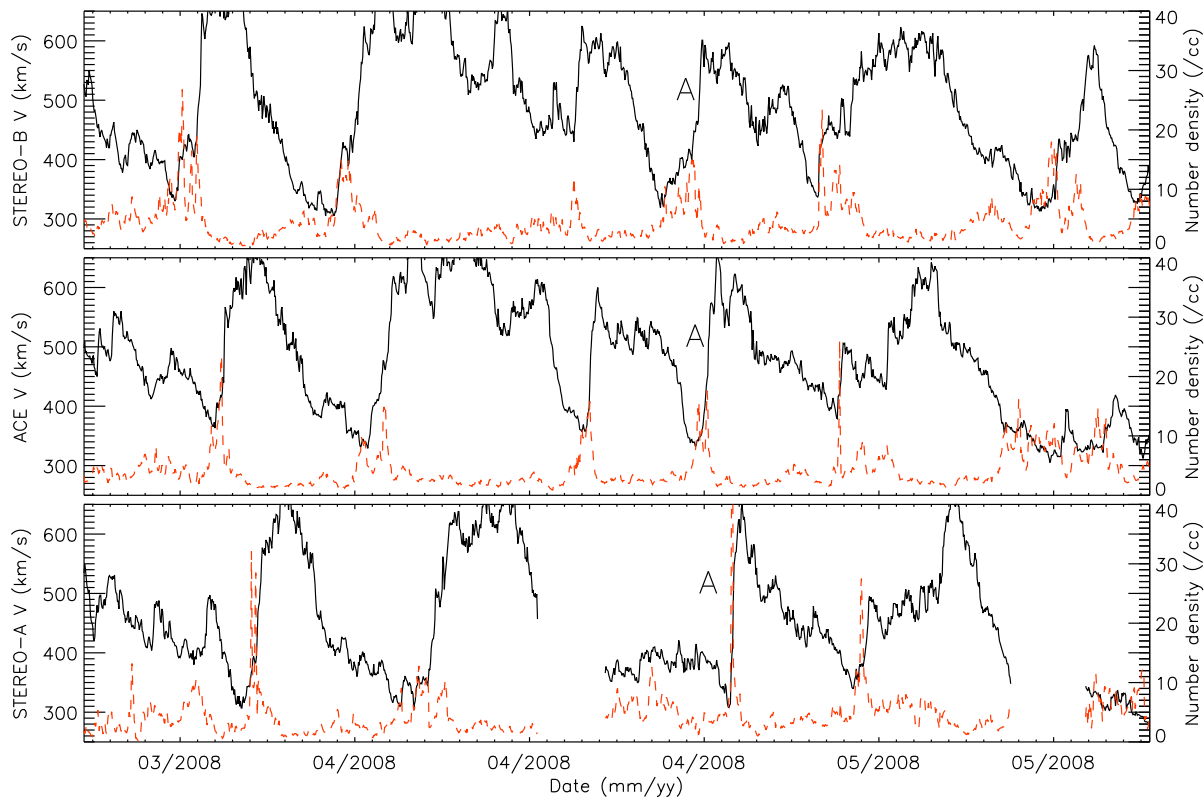

Figure 10 This figure highlights an event observed in-situ by each of the spacecraft, showing the speed (black) and density (red-dashed) data. The gradual development of the material to look increasingly shocked (i.e. the density enhancement sharpening) can be seen in a couple of the events shown here, for example that event labelled A. It can be seen that as the feature develops over time (i.e. from STEREO-B to ACE to STEREO-A) the velocity enhancement becomes sharper and the density enhancement much narrower.

The analysis that we conducted in order to estimate the propagation speed of the transients in this study assumed that the material had constant properties while it was being observed, for each blob over its lifetime of observation and for every blob associated with the same CIR, i.e. it assumed that as the Sun rotated, the material that it ejected would be at a constant speed. Figures 10 and 11 highlight particular features of the previous plots. Looking at these reveal that there is actually a great deal of variability in both speed and density data from one spacecraft to the next and one Carrington rotation to the next. The speed measured by STEREO-B, for example, in the second half of 2008, shows a pair of peaks that recur with a period of a Carrington rotation; however, the solar-wind streams can be seen to merge/diverge on time scales shorter than a Carrington period. Thus, there is variability on the time scale of a Carrington rotation, i.e. approximately 27 days. During this period, a pair of features of note have been labelled as B0 and B1. As seen by STEREO-B, B0 is a speed enhancement with a smaller shoulder-like structure at a lower speed labelled B1. If STEREO-B in-situ data alone were being considered, the two would likely not be interpreted as different features, but having data from multiple spacecraft indicates otherwise. Considering now the same features as observed at ACE, the "shoulder" on B0 has become less pronounced, and instead the speed of the fast solar-wind stream that arrived behind it appears with an enhanced speed, being now equal to the speed of the feature previously labelled B1. Thus, it appears that there are two streams moving at differing speeds, and they are separating out as time progresses. Looking now at the feature seen by STEREO-A, we appear to be observing B1 merging with another fast solar-wind stream. It can be seen that 


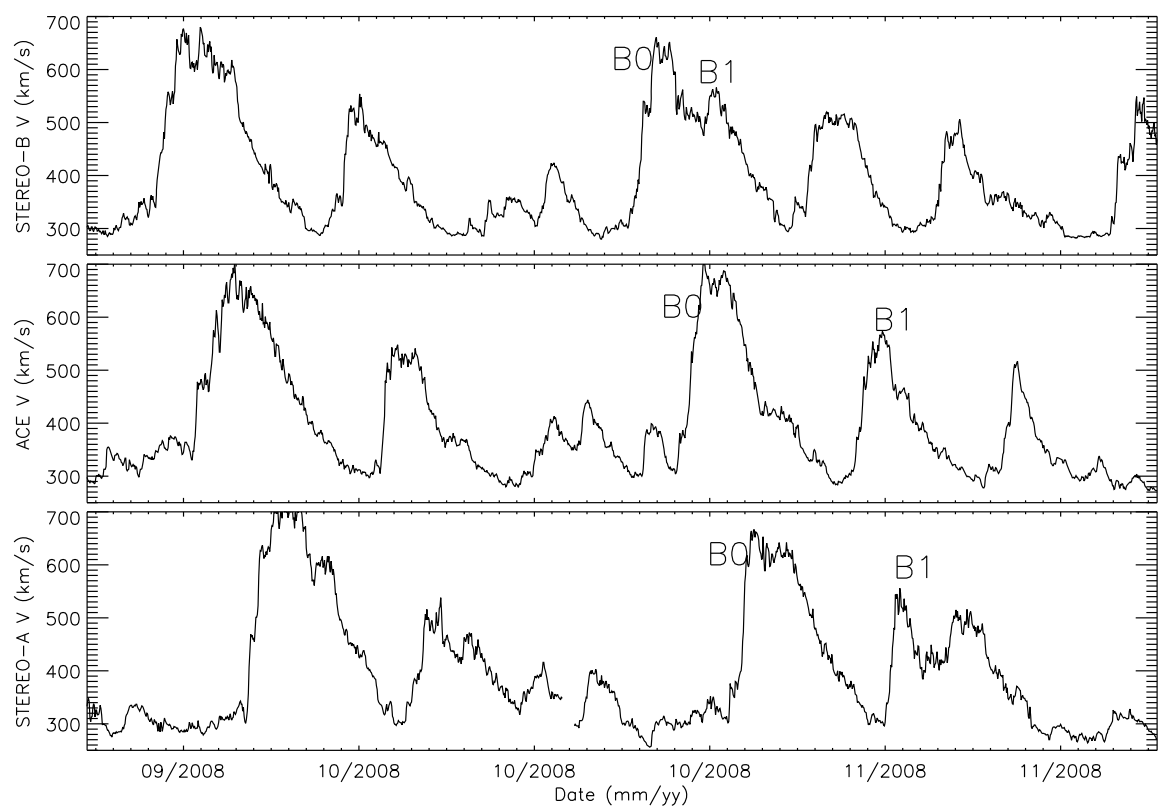

Figure 11 Another in-situ feature seen by each of the STEREO and ACE spacecraft, showing a pair of solar-wind streams moving at different speeds, indicated by B0 and B1. These two streams can be seen to separate with time (going from STEREO-B to ACE and then to STEREO-A) and so display variability that calls into question the validity of some of the assumptions often made when analysing solar-wind transients observed by STEREO/HI.

there is therefore significant variability between observations from each of the spacecraft. Looking now at another feature, labelled "A" in each of the plots, we can see that the speed profile indicates a fast solar-wind stream, the leading edge of which over time (looking from STEREO-B to ACE to STEREO-A) appears to sharpen dramatically, looking increasingly like shocked solar wind. Comparing this with the density data, we see a density enhancement narrow with time, slowly increasing in maximum density, and then dramatically sharpening and increasing in density when seen at STEREO-A, once again looking more like a shocked structure. Both of these examples serve to illustrate that there is significant variability comparing data from one spacecraft to the next. Considering the rotation rate of the Sun, and the longitudinal separation between the spacecraft, this means that there is variability on the time scale of 1.5-2 days. CIR observations at different spacecraft have been compared by Mason et al. (2009) and more specifically in reference to STEREO observations (Leske et al., 2008) and Wind observations (Sanderson et al., 1998). These authors also noted that features present in in-situ data from one spacecraft are not always observed when one would expect at another spacecraft with an angular separation, as noticed here. They point out that one major factor that causes this is that the Ecliptic plane, in which these observations are made, does not lie along the plane containing the solar Equator. All of this indicates that one should be quite careful when making approximations about constant solar-wind conditions, although, as already mentioned, despite this the predicted arrival times established from HI observations make such approximations appear to form a good match to the in-situ data on the time scales presented here. 
Figure 12 The top panel shows the superposed-epoch analysis using the ACE in-situ data. Speed is in black and density in blue. The middle and lower panels show this for STEREO-A and STEREO-B in-situ data. In each case, it shows the superposedepoch analysis conducted on all 40 events mentioned previously in the article, with the red line corresponding to the predicted arrival time of the events at each of the spacecraft. We can see that it has been possible to identify CIRs in HI that, when extrapolated to each of the three spacecraft, match the in-situ signature we would expect from Figure 1.
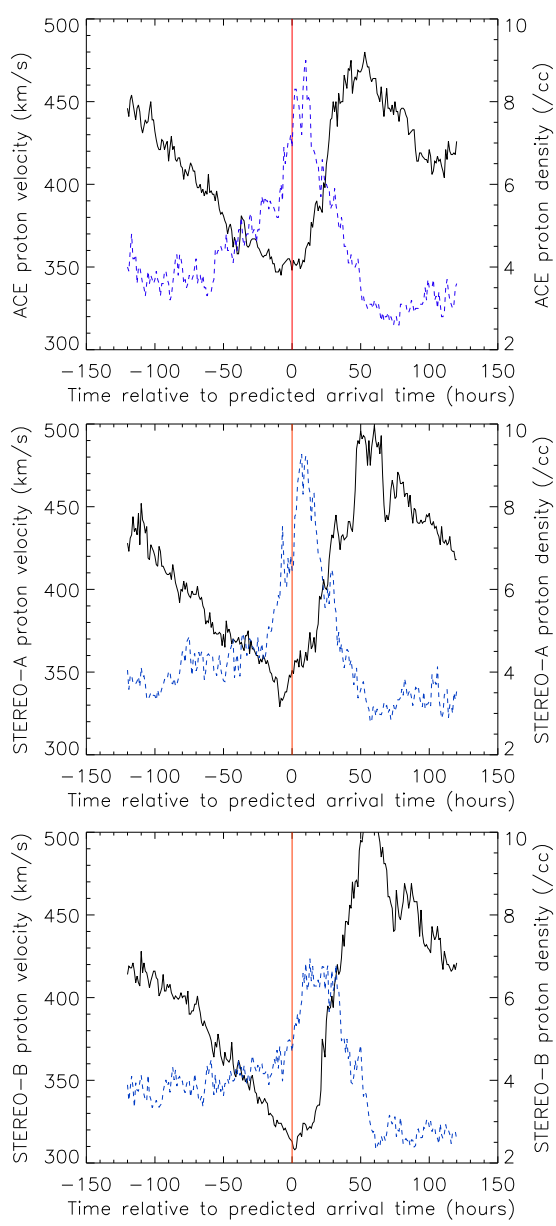

\section{Superposed-Epoch Analysis}

A superposed-epoch study of the 40 CIR events characterised in the STEREO-A/HI data was conducted using the HI-predicted arrival times as the zero time, using hourly ACE and STEREO data. The top panel of Figure 12 presents the results of the superposed-epoch analysis, showing the average values of bulk proton speed (black) and proton density (blue) from ACE, and the middle and bottom panels show the same but using in-situ data from the STEREO-A (middle) and -B (bottom) spacecraft. In each case, the epoch zero time is shown by a red-vertical line. Each plot illustrates the speed and density signatures typical of a CIR, with the denser material also being some of the slower material. In a similar superposed-epoch analysis conducted by Davis et al. (2012) it appears that the denser material that should correspond to that observed by STEREO/HI is not quite associated with the slow solar wind, but rather with material travelling at a speed slightly higher than that. In the plots presented here, there is perhaps the indication that the denser material might correspond to an intermediate speed in the STEREO-B plot, but this is not at all clear in the STEREO-A and ACE plots (Figure 12). As the plots that Davis et al. (2012) present are created from OMNI data, it is this last instance that we would expect to agree most closely. 
On balance from this article, it appears that these CIR-associated transients appear to be travelling at, or perhaps very close to, the slow solar-wind speed, in agreement with Sheeley et al. (1997), although the latter were not specifically looking at CIR entrained blobs. The standard deviations associated with the data presented in Figure 12 are $\sigma_{V} \approx 150 \mathrm{~km} \mathrm{~s}^{-1}$, $\sigma_{\rho} \approx 6 \mathrm{~cm}^{-3}$.

\section{Discussion}

In this article, we have identified solar-wind transient features associated with CIRs in STEREO/HI during a period of low solar activity, when such features should be more distinct, confirming the signatures of CIRs seen in STEREO/HI in the process. We have analysed the propagation characteristics of a significant number of these features and been able to improve the estimates or propagation speed over some previous studies. We have been able to confirm that the CIR-related transients travel at, or close to, the slow solar-wind speed. There are, of course, some factors to keep in mind when considering these results.

From the observations presented in the previous sections, it is apparent that during the period under study, solar-wind source regions on the solar surface can last for multiple solarrotation periods; however, a given source does not eject material with constant properties on time scales of even a few days, in agreement with Mason et al. (2009). This suggests possible sources of error in solar-wind analysis techniques that assume a constant propagation speed over the entire CIR. The fitting technique that we have used in this study will be particularly susceptible to this, as we are attempting to simultaneously fit multiple time-elongation profiles that will in reality (with previously mentioned speed variations on time scales of one or two days) actually be travelling at different speeds. Perhaps by relaxing some of these assumptions, it might be possible to improve the reliability with which transient arrival times at 1 AU can be ascertained, thereby improving space-weather predictive capabilities. Work has been conducted by Lugaz and Kintner (2013) investigating the effects of drag on solar-wind transients, and Sheeley et al. (1997) have considered acceleration through a coronagraph field of view, and if this effect could be folded into estimates of solar-wind transients seen in STEREO/HI results, this might be further improved.

It has been seen that rotation after rotation, a given source region appears to drift across the surface of the Sun. This could be a real effect; however, it is more likely that the rotation rate being used to represent these source regions is incorrect. Assuming that the only factor affecting this rotation rate is the latitude of the source region, then it should be possible to ascribe each source region its own individual rotation rate. However, it does not appear that ascribing an average solar-rotation period (as we have done) is reliable when considering time scales of a solar-rotation period. To establish a more exact rotation rate, images of the solar disc taken from, e.g. STEREO, the Solar and Heliospheric Observatory (SOHO), and the Solar Dynamics Observatory (SDO) could be used to find the position (and hence latitude) of the source region.

\section{Conclusion}

Having established evidence for CIR-related solar-wind transients in STEREO/HI-A J-maps and identified and analysed such features from 2007 - mid-2010, we found that it was possible to improve the estimates of their kinematic properties. It was found that fast solar-wind 
source regions can persist for multiple solar rotations; however, the properties of the material ejected from them are not a constant over these time scales and can vary noticeably over a matter of days. It was thus suggested that a more thorough examination of the transient acceleration profiles might allow for better fitting. It was noted that it is difficult to find a characteristic solar-rotation rate for these source regions and the assumption that some standard rotation rate can be used for multiple source regions is not necessarily valid, but this could be improved by using images of the solar disc to find a location of a source region on the Sun and use this to estimate its location and thus its particular rotation rate. By considering a significant number of CIR-related features, it was confirmed that solar-wind transients associated with CIRs seem to travel at or very close to the slow solar-wind speed.

Acknowledgements The authors would like to thank the Science and Technology Facilities Council (STFC) and United Kingdom Space Agency (UKSA) for making this research possible, along with the STEREO team and the UK Solar System Data Centre (UKSSDC). T.M. Conlon and A.O. Williams were supported by an STFC, UK studentship and S.E. Milan was supported by STFC grant ST/K001000/1.

Disclosure of Potential Conflicts of Interest The authors declare that they have no conflicts of interest.

Open Access This article is distributed under the terms of the Creative Commons Attribution 4.0 International License (http://creativecommons.org/licenses/by/4.0/), which permits unrestricted use, distribution, and reproduction in any medium, provided you give appropriate credit to the original author(s) and the source, provide a link to the Creative Commons license, and indicate if changes were made.

\section{References}

Acuña, M.H., Curtis, D., Scheifele, J.L., Russell, C.T., Schroeder, P., Szabo, A., Luhmann, J.G.: 2008, The STEREO/IMPACT magnetic field experiment. Space Sci. Rev. 136(1-4), 203. DOI.

Borovsky, J.E., Denton, M.H.: 2006, Differences between CME-driven storms and CIR-driven storms. J. Geophys. Res. 111(A7), A07S08. DOI.

Conlon, T.M., Milan, S.E., Davies, J.A.: 2014, Assessing the effect of spacecraft motion on single-spacecraft solar wind tracking techniques. Solar Phys. 289, 3935. DOI.

Davies, J.A., Harrison, R.A., Rouillard, A.P., Sheeley, N.R., Perry, C.H., Bewsher, D., Davis, C.J., Eyles, C.J., Crothers, S.R., Brown, D.S.: 2009, A synoptic view of solar transient evolution in the inner heliosphere using the Heliospheric Imagers on STEREO. Geophys. Res. Lett. 36 L08102. DOI.

Davies, J.A., Perry, C.H., Trines, R.M.G.M., Harrison, R.A., Lugaz, N., Möstl, C., Liu, Y.D., Steed, K.: 2013, Establishing a stereoscopic technique for determining the kinematic properties of solar wind transients based on a generalized self-similarly expanding circular geometry. Astrophys. J. 777, 167. DOI.

Davis, C.J., Davies, J.a., Owens, M.J., Lockwood, M.: 2012, Predicting the arrival of high-speed solar wind streams at Earth using the STEREO Heliospheric Imagers. Space Weather 10 S02003. DOI.

Eyles, C.J., Simnett, G.M., Cooke, M.P., Jackson, B.V., Buffington, A., Hick, P.P., Waltham, N.R., King, J.M., Anderson, P.A., Holladay, P.E.: 2003, The Solar Mass Ejection Imager (SMEI). Solar Phys. 217(2), 319. DOI.

Eyles, C.J., Harrison, R.A., Davis, C.J., Waltham, N.R., Shaughnessy, B.M., Mapson-Menard, H.C.A., Bewsher, D., Crothers, S.R., Davies, J.A., Simnett, G.M., Howard, R.A., Moses, J.D., Newmark, J.S., Socker, D.G., Halain, J.-P., Defise, J.-M., Mazy, E., Rochus, P.: 2009, The Heliospheric Imagers onboard the STEREO mission. Solar Phys. 254(2), 387. DOI.

Gosling, J.T., Pizzo, V.J.: 1999, Formation and evolution of corotating interaction regions and their three dimensional structure. Space Sci. Rev. 89, 21. DOI.

Howard, T.A., Tappin, S.J.: 2009, Interplanetary coronal mass ejections observed in the heliosphere: 1 . Review of theory. Space Sci. Rev. 147(1-2), 31. DOI.

Howard, T.A., Webb, D.F., Tappin, S.J., Mizuno, D.R., Johnston, J.C.: 2006, Tracking halo coronal mass ejections from 0-1 AU and space weather forecasting using the Solar Mass Ejection Imager (SMEI). J. Geophys. Res. 111(A4), A04105. DOI.

Howard, R.A., Moses, J.D., Vourlidas, A., Newmark, J.S., Socker, D.G., Plunkett, S.P., Korendyke, C.M., Cook, J.W., Hurley, A., Davila, J.M., Thompson, W.T., St Cyr, O.C., Mentzell, E., Mehalick, K., Lemen, J.R., Wuelser, J.P., Duncan, D.W., Tarbell, T.D., Wolfson, C.J., Moore, A., Harrison, R.A., Waltham, 
N.R., Lang, J., Davis, C.J., Eyles, C.J., Mapson-Menard, H., Simnett, G.M., Halain, J.P., Defise, J.M., Mazy, E., Rochus, P., Mercier, R., Ravet, M.F., Delmotte, F., Auchere, F., Delaboudiniere, J.P., Bothmer, V., Deutsch, W., Wang, D., Rich, N., Cooper, S., Stephens, V., Maahs, G., Baugh, R., McMullin, D., Carter, T.: 2008, Sun Earth Connection Coronal and Heliospheric Investigation (SECCHI). Space Sci. Rev. 136(1-4), 67. DOI.

Kahler, S.W., Webb, D.F.: 2007, V arc interplanetary coronal mass ejections observed with the Solar Mass Ejection Imager. J. Geophys. Res. 112. A09103 DOI.

Kaiser, M.L., Kucera, T.A., Davila, J.M., Cyr, O.C., Guhathakurta, M., Christian, E.: 2007, The STEREO mission: an introduction. Space Sci. Rev. 136(1-4), 5. DOI.

Lang, K.R.: 2001, The Cambridge Encyclopedia of the Sun, Cambridge University Press, Cambridge. 0521780934.

Leske, R.A., Mewaldt, R.A., Mason, G.M., Cohen, C.M.S., Cummings, A.C., Davis, A.J., Labrador, A.W., Miyasaka, H., Stone, E.C., Wiedenbeck, M.E.: 2008, Particle acceleration and transport in the heliosphere and beyond. In: Li, G., Hu, Q., Verkhoglyadova, O., Zank, G., Lin, R.P., Luhmann, J. (eds.) 7th International Astrophysics Conference, AIP Conf. Proc., American Institute of Physics, New York.

Lugaz, N., Kintner, P.: 2013, Effect of solar wind drag on the determination of the properties of coronal mass ejections from Heliospheric Images. Solar Phys. 285(1-2), 281. DOI.

Lugaz, N., Vourlidas, A., Roussev, I.I.: 2009, Deriving the radial distances of wide coronal mass ejections from elongation measurements in the heliosphere - application to CME-CME interaction. Ann. Geophys. 27(9), 3479.

Mason, G.M., Desai, M.I., Mall, U., Korth, A., Bucik, R., Rosenvinge, T.T., Simunac, K.D.: 2009, In situ observations of CIRs on STEREO, wind, and ACE during 2007-2008. Solar Phys. 256(1-2), 393. DOI.

Poletto, G.: 2013, Sources of solar wind over the solar activity cycle. J. Advert. Res. 4(3), 215. DOI.

Rouillard, A.P., Davies, J.A., Forsyth, R.J., Rees, A., Davis, C.J., Harrison, R.A., Lockwood, M., Bewsher, D., Crothers, S.R., Eyles, C.J., Hapgood, M., Perry, C.H.: 2008, First imaging of corotating interaction regions using the STEREO spacecraft. Geophys. Res. Lett. 35 L10110. DOI.

Rouillard, A.P., Savani, N.P., Davies, J.A., Lavraud, B., Forsyth, R.J., Morley, S.K., Opitz, A., Sheeley, N.R., Burlaga, L.F., Sauvaud, J.-A., Simunac, K.D.C., Luhmann, J.G., Galvin, A.B., Crothers, S.R., Davis, C.J., Harrison, R.A., Lockwood, M., Eyles, C.J., Bewsher, D., Brown, D.S.: 2009, A multispacecraft analysis of a small-scale transient entrained by solar wind streams. Solar Phys. 256(1-2), 307. DOI.

Rouillard, A.P., Davies, J.A., Lavraud, B., Forsyth, R.J., Savani, N.P., Bewsher, D., Brown, D.S., Sheeley, N.R., Davis, C.J., Harrison, R.A., Howard, R.A., Vourlidas, A., Lockwood, M., Crothers, S.R., Eyles, C.J.: 2010, Intermittent release of transients in the slow solar wind: 1. Remote sensing observations. J. Geophys. Res. 115 A04103. DOI.

Sanderson, T.R., Lin, R.P., Larson, D.E., Mccarthy, M.P., Parks, G.K., Lormant, N., Lepping, K.O.R.P., Steinberg, J.T., Hoeksema, J.T.: 1998, Wind observations of the influence of the Sun's magnetic field on the interplanetary medium at 1 AU. J. Geophys. Res. 103(A8), 17235. DOI.

Sheeley, N.R., Rouillard, A.P.: 2010, Tracking streamer blobs into the heliosphere. Astrophys. J. 715(1), 300. DOI.

Sheeley, N.R., Wang, Y.M., Hawley, S.H., Brueckner, G.E., Dere, K.P., Howard, R.A., Koomen, M.J., Korendyke, C.M., Michels, D.J., Paswaters, S.E., Socker, D.G., Lamy, P.L., Llebaria, A.: 1997, Measurements of flow speeds in the corona between 2 and 30 R. Astrophys. J. 484, 472. DOI.

Sheeley, N.R., Walters, J.H., Wang, Y.-M., Howard, R.A.: 1999, Continuous tracking of coronal outflows: two kinds of coronal mass ejections. J. Geophys. Res. 104, 24739. DOI.

Sheeley, N.R., Herbst, A.D., Palatchi, C.A., Wang, Y.M., Howard, R.A., Moses, J.D., Vourlidas, A., Newmark, J.S., Socker, D.G., Plunkett, S.P., Korendyke, C.M., Burlaga, L.F., Davila, J.M., Thompson, W.T., St Cyr, O.C., Harrison, R.A., Davis, C.J., Eyles, C.J., Halain, J.P., Wang, D., Rich, N.B., Battams, K., Esfandiari, E., Stenborg, G.: 2008, Heliospheric images of the solar wind at Earth. Astrophys. J. 675(1), 853. DOI.

Smith, E.J., Wolfe, J.H.: 1976, Observations of interaction regions and corotating shocks between one and five AU: Pioners 10 and 11. Geophys. Res. Lett. 3(3) 137. DOI.

Tappin, S.J., Howard, T.a.: 2009, Direct observation of a corotating interaction region by three spacecraft. Astrophys. J. 702(2), 862. DOI.

Williams, A.O., Davies, J.A., Milan, S.E., Rouillard, A.P., Davis, C.J., Perry, C.H., Harrison, R.A.: 2009, Deriving solar transient characteristics from single spacecraft STEREO/HI elongation variations: a theoretical assessment of the technique. Ann. Geophys. 27(1), 4359.

Williams, A.O., Edberg, N.J.T., Milan, S.E., Lester, M., Fränz, M., Davies, J.A.: 2011, Tracking corotating interaction regions from the Sun through to the orbit of Mars using ACE, MEX, VEX, and STEREO. J. Geophys. Res. 116 A08103. DOI. 